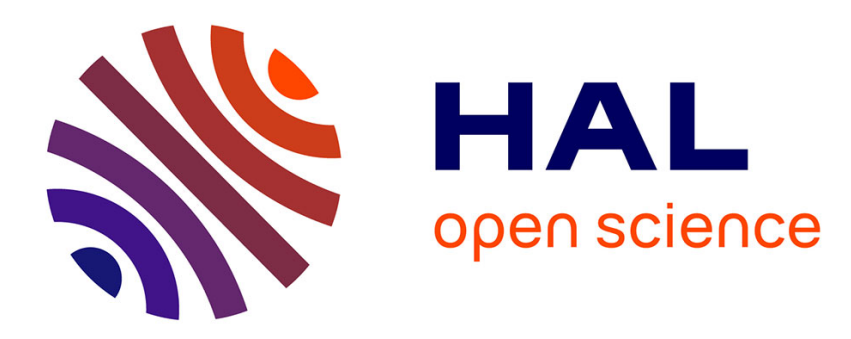

\title{
Les besoins de supraconducteurs pour la fusion thermonucléaire
}

\author{
J. Parain
}

\section{To cite this version:}

J. Parain. Les besoins de supraconducteurs pour la fusion thermonucléaire. Revue de Physique Appliquée, 1977, 12 (8), pp.1111-1119. 10.1051/rphysap:019770012080111100 . jpa-00244287

\section{HAL Id: jpa-00244287 https://hal.science/jpa-00244287}

Submitted on 1 Jan 1977

HAL is a multi-disciplinary open access archive for the deposit and dissemination of scientific research documents, whether they are published or not. The documents may come from teaching and research institutions in France or abroad, or from public or private research centers.
L'archive ouverte pluridisciplinaire HAL, est destinée au dépôt et à la diffusion de documents scientifiques de niveau recherche, publiés ou non, émanant des établissements d'enseignement et de recherche français ou étrangers, des laboratoires publics ou privés. 


\author{
Classification \\ Physics Abstracts \\ $6.424-4.640-8.450$
}

\title{
LES BESOINS DE SUPRACONDUCTEURS POUR LA FUSION THERMONUCLÉAIRE $(*)$
}

\author{
J. PARAIN \\ DPH/PE-STIPE, C. E. N. de Saclay, B. P. no 2, 91190 Gif-sur-Yvette, France
}

(Reçu le 28 décembre 1976, accepté le 20 avril 1977)

\begin{abstract}
Résumé. - Dans une première partie, on décrit les systèmes magnétiques des projets de grands TOKAMAKS. L'utilisation des supraconducteurs est devenue indispensable dès la prochaine génération de TOKAMAKS. On situe les problèmes rencontrés dans ces projets par rapport à l'état actuel de la technologie des supraconducteurs.

Dans une seconde partie, la description de l'étude de la version supraconductrice d'un TOKAMAK conduit à préciser les problèmes, principalement ceux rencontrés dans l'étude du conducteur.

Abstract. - In a first part, we describe the magnetic system for large TOKAMAKS. The use of superconducting coils will become essential for the next generation of TOKAMAKS. We try to estimate the problems raised by these projects in the present state of superconducting magnet technology.

In a second part, we describe in detail a superconducting version of a TOKAMAK and in particular we discuss the problems encountered with the conductor design.
\end{abstract}

Des nouveaux matériaux niobium-titane $(\mathrm{Nb}-\mathrm{Ti})$ ou niobium-étain $\left(\mathrm{Nb}_{3} \mathrm{Sn}\right)$ sont supraconducteurs à la température de l'hélium liquide 4,2 K. De plus, ces matériaux permettent des densités de courant très supérieures à celles obtenues dans le cuivre et conservent leurs propriétés de supraconducteur pour des inductions pouvant atteindre 10 à $15 \mathrm{~T}$. Ces supraconducteurs sont, soit déjà utilisés, soit envisagés pour toutes les expériences où un plasma est confiné magnétiquement. Nous regarderons leur utilisation aux machines du type TOKAMAK qui semble la voie la plus sûre vers la fusion thermonucléaire [1].

1. Les projets et les études des grands TOKAMAKS. Le système magnétique d'un TOKAMAK est constitué de deux types de bobines. D'une part, un ensemble de bobines disposées sur un tore produit le champ dit toroïdal ; ce champ est fixe pour les besoins de l'expérience, il est pulsé dans le cas de l'utilisation de bobinages de cuivre pour réduire la puissance dépensée. D'autre part, le champ poloïdal produit par des bobines circulaires centrées sur l'axe du tore assure la stabilité et l'échauffement du plasma. Ces bobines sont pulsées, leur champ s'établit en même temps que le courant plasma. La figure 1 montre schématiquement la disposition des différentes bobines d'un TOKAMAK.

(*) Communication présentée au Congrès National de Physique des Plasmas, Paris, 6-10 décembre 1976.

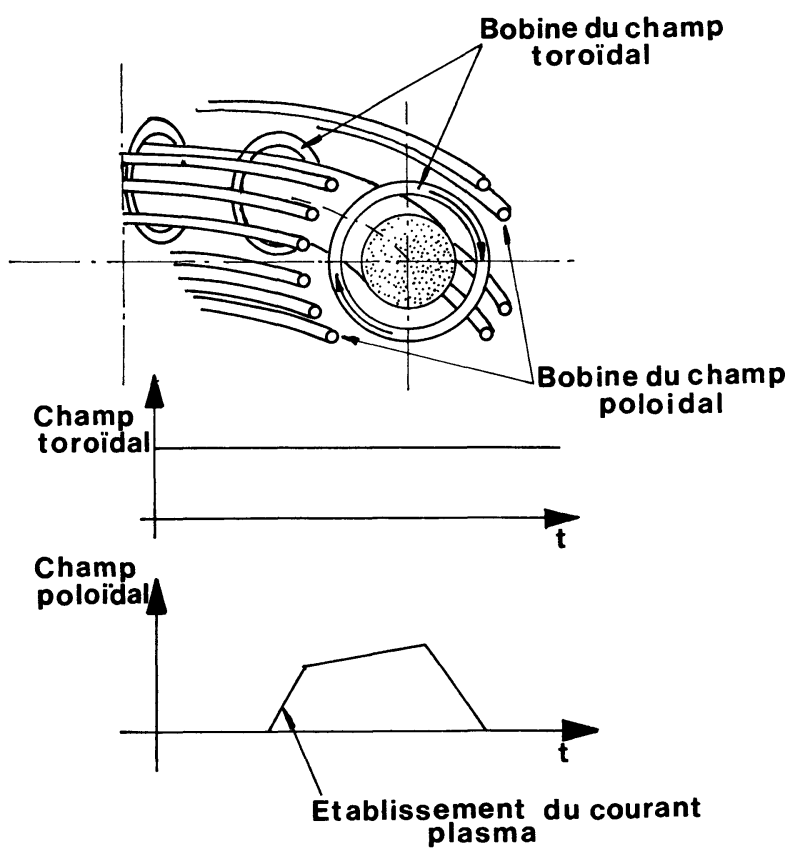

Fig. 1. - Vue schématique des différentes bobines et variation des champs en fonction du temps.

[Schematic view of the coils and magnetic fields versus the time.]

Le tableau suivant donne les étapes successives de la réalisation des grands TOKAMAKS. Le premier projet cité est le J. E. T. (Joint European Torus) dont la mise en fonctionnement est prévue pour 1980. L'étude 


\section{TABLEAU I}

Grand rayon du plasma

Petit rayon du plasma

Champ toroïdal

Courant plasma

Energie stockée

Temps d'établissement du plasma

Temps de palier

Temps de répétition (m)

(m)

(T)

(MA)

(GJ)

(s)

(s)

(s)
J. E. T.

3,0

1,25

3,4

4,8

1,4

1,0

20

630
T. E. P. R.

6,25

2,1

3,6

6,0

30

5

50

75
U. W. M. A. K. II

13

5

3,6

15

220

10

5400

5730
T. E. P. R. (Tokamak Experimental Power Reactor) remplit les conditions permettant de fournir de la puissance électrique. L'étude U. W. M. A. K. est la description d'un réacteur de fusion de $5 \mathrm{GW}$ thermiques.

Ces trois études sont prises à titre d'exemple; la première est européenne, la deuxième et la troisième ont été étudiées aux Etats-Unis. Des études similaires existent en U. R. S. S. ou au Japon.

En annexe, on montre comment varient les caractéristiques magnétiques de cette classe de TOKAMAK.

Les champs magnétiques du J. E. T. sont obtenus avec des bobines conventionnelles en cuivre, ce qui impose un faible facteur d'utilisation, le champ toroïdal étant pulsé. Les deux autres études citées utilisent impérativement des bobinages supraconducteurs.

Dans le cas de l'étude U. W. M. A. K. II, une puissance électrique de plusieurs gigawatts serait nécessaire pour la production du champ toroïdal dans le cas de l'utilisation de bobines de cuivre.

En U. R. S. S., un TOKAMAK T7 est en cours de construction. Il utilise des bobinages supraconducteurs bien qu'il soit plus petit que les projets considérés dans le tableau $\mathrm{I}$. (Le grand rayon est de $1,2 \mathrm{~m}$, le rayon plasma de 0,35 m et l'induction de 3,5 T.) Cette réalisation donnera des résultats significatifs sur l'utilisation des supraconducteurs [2].

2. Situation de la technologie. - On donne dans ce chapitre les buts à obtenir pour la construction des grands TOKAMAKS et l'état des réalisations significatives dans le domaine des supraconducteurs.

Le premier problème que l'on rencontre est la valeur maximale du champ que l'on peut réaliser avec des matériaux supraconducteurs.

La figure 2 donne la densité de courant critique, c'est-à-dire le courant pour lequel le matériau perd son état supraconducteur, en fonction de l'induction. Sur la même figure sont portées les caractéristiques du Nb-Ti et $\mathrm{du} \mathrm{Nb}_{3} \mathrm{Sn}$. Ce dernier conducteur est plus intéressant, la densité de courant et le champ sont plus élevés, mais le $\mathrm{Nb}_{3} \mathrm{Sn}$ supporte mal les contraintes et il n'a été utilisé à ce jour que pour réaliser des petites bobines. Cependant, des études de TOKAMAK sont faites en utilisant des valeurs maximales de champ magnétique de $16 \mathrm{~T}$. Il est, en fait, intéressant d'anticiper les propriétés du $\mathrm{Nb}_{3} \mathrm{Sn}$, parallèlement de faire l'effort

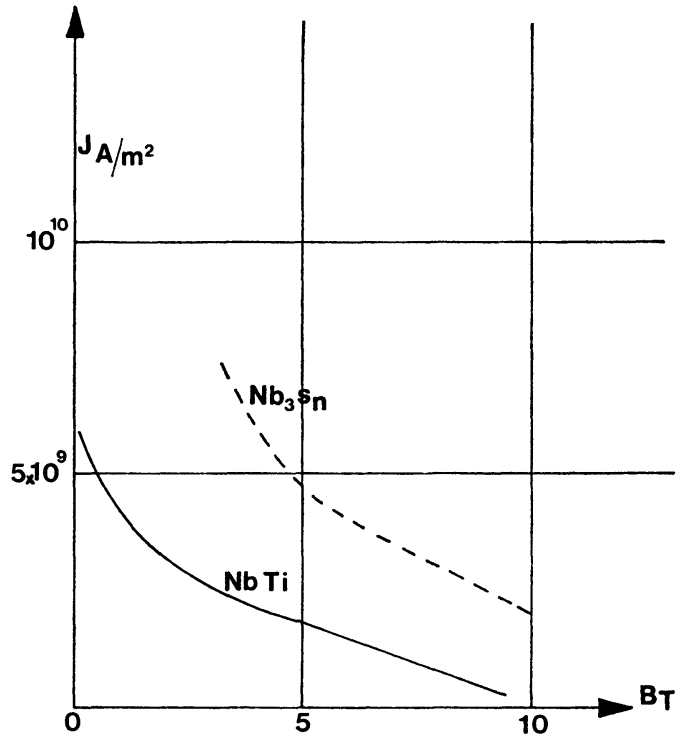

Fig. 2. - Densité de courant en fonction de l'induction pour le niobium-titane ( $\mathrm{Nb}-\mathrm{Ti})$ et le niobium-étain $\left(\mathrm{Nb}_{3} \mathrm{Sn}\right)$ à la température de $4,2 \mathrm{~K}$.

[Current density versus the magnetic field for niobium-titanium $(\mathrm{Nb}-\mathrm{Ti})$ and niobium-tin $\left(\mathrm{Nb}_{3} \mathrm{Sn}\right)$ at $4.2 \mathrm{~K}$.]

nécessaire pour sa mise au point et de dégager les problèmes posés par l'utilisation des champs forts dans les grands TOKÁMAKS. A ce jour, seul le NbTi est utilisé pour les grandes réalisations.

Il faut remarquer que le champ donné dans le tableau I est le champ sur l'axe du plasma. A cause de la géométrie torique la valeur maximale du champ sur le conducteur des bobines dépasse $8 \mathrm{~T}$ dans les projets décrits, ce qui oblige à utiliser le $\mathrm{Nb}$ - $\mathrm{Ti}$ aux limites de ses performances. Le problème devient plus difficile pour la réalisation des petits tores qui exigent une grande densité de courant, comme montré dans l'annexe.

La densité de courant portée sur la figure 2 est la densité dans le supraconducteur; en fait, le supraconducteur a un comportement instable, à un échauffement accidentel correspond une diminution du courant critique et la recherche de la stabilité diminue considérablement ces densités de courant.

Les grandes réalisations ont utilisé des conducteurs stabilisés cryostatiquement. Le matériau supraconduc- 
teur est placé dans une matrice d'un métal ayant une bonne conductibilité thermique et électrique. Cette matrice doit, de plus, permettre un bon échange thermique avec le réfrigérant hélium liquide ou gazeux. Dans l'hypothèse où le matériau passe de l'état supraconducteur à l'état normal, le courant circule partiellement dans la matrice de cuivre ou d'aluminium et les échanges doivent être tels que la température du supraconducteur reste inférieure à sa température critique. Cette méthode de stabilisation a l'inconvénient de conduire à des densités de courant dans le conducteur (supraconducteur plus stabilisant faibles), 10 à $20 \mathrm{~A} / \mathrm{mm}^{2}$ dans le cas des inductions considérées.

Pour des réalisations nécessitant une forte densité de courant, la stabilité est obtenue d'une part, en faisant jouer le rôle de stabilisant à la matrice de métal et, d'autre part, en utilisant le supraconducteur sous forme de filaments fins de diamètre inférieur à $50 \mu$. Les densités de courant obtenues sont beaucoup plus élevées que dans le cas de l'utilisation de la stabilité cryostatique ; elles peuvent atteindre 50 à $100 \mathrm{~A} / \mathrm{mm}^{2}$ dans le cas des inductions considérées.

La figure 3 montre la coupe d'un conducteur de $\mathrm{Nb}$ Ti de $1,3 \mathrm{~mm}$. Ce composite sera assemblé pour réaliser le conducteur final. La densité de courant apparente dans ce composite est de $300 \mathrm{~A} / \mathrm{mm}^{2}$ à $5,5 \mathrm{~T}$.

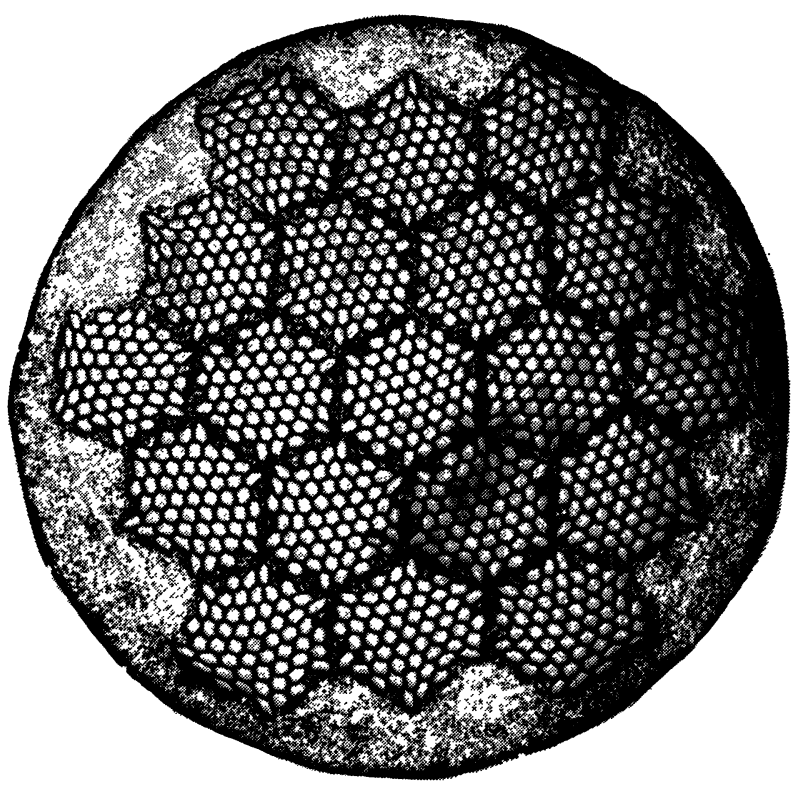

Fig. 3. - Conducteur à 1045 filaments de $10 \mu$ de Nb-Ti dans une matrice de cuivre. La dimension extérieure du conducteur est de $1,3 \mathrm{~mm}$.

[Conductor with $104510 \mu$ filaments of $\mathrm{Nb}$-Ti in a copper matrix. The external size of the conductor is $1.3 \mathrm{~mm}$.]

Le second problème que l'on rencontre dans la réalisation du système magnétique d'un TOKAMAK est dû aux pertes dans le supraconducteur provoquées par la variation du champ poloïdal. Ce champ atteint $0,4 \mathrm{~T}$ au niveau des bobines du champ toroïdal. Cette valeur est indépendante de la taille dans la classe de TOKAMAK décrit en annexe. Ce champ s'établit en une fraction de seconde pour les petits TOKAMAKS, ou en quelques secondes pour les TOKAMAKS présentés dans le tableau I.

La variation du champ poloïdal conditionne très fortement le système magnétique des TOKAMAKS. De plus, la destruction accidentelle du plasma provoque une rapide variation du champ magnétique au niveau des bobines. Cette rupture du courant plasma pourra être certainement contrôlée pour les grands TOKAMAKS ; elle reste un important problème aujourd'hui.

Le matériau supraconducteur utilisé n'a pas un parfait diamagnétisme; il y a pénétration du champ magnétique d'où dissipation d'énergie pendant les variations de champ. Ces pertes par magnétisation dépendent de la direction du champ variable par rapport au conducteur. Dans le cas où le champ variable est perpendiculaire au conducteur, la densité des pertes est de la forme :

$$
W=2 d J_{\mathrm{c}} \eta \Delta B
$$

où $d$ est le diamètre des filaments, $\eta$ le taux de remplissage dans le composite, $J_{c}$ la densité de courant critique axial et $\Delta B$ l'amplitude du champ variable. Dans le cas où le champ variable est parallèle au conducteur, l'expression des pertes est différente ; elle fait notamment intervenir la densité de courant critique azimutal [4].

Le conducteur est réalisé par assemblage des composites dans une matrice de cuivre pour obtenir un conducteur massif ou par câblage ; dans les deux cas s'ajoutent des pertes par courant de circulation entre les brins ou entre les composites, ou dans la matrice de cuivre. Ces pertes sont du type courant de Foucault : elles sont proportionnelles à $\Delta B^{2} / T$ où $\Delta B$ est l'amplitude du champ et $T$ sa constante de temps.

L'utilisation d'un câble permet d'éviter les pertes dans la matrice de cuivre qui se produisent dans le conducteur massif ; mais l'isolement entre les composites est nécessaire ; cet isolement peut être obtenu par oxydation ou par l'utilisation de barrières de cupronickel. Pour les grands TOKAMAKS la durée de la variation du champ poloïdal est estimée à $10 \mathrm{~s}$ et l'utilisation d'un conducteur massif semble encore possible.

Ces pertes dans le conducteur sont dissipées à la température de l'hélium liquide ; elles sont donc très onéreuses à compenser mais, de plus, elles élèvent la température du supraconducteur et peuvent amener l'ensemble du bobinage à retourner à l'état normal.

L'utilisation d'un écran peut éviter les effets de la variation du champ poloïdal et ceux de la rupture du plasma ; l'écran doit entourer les bobines produisant le champ toroïdal [3]. Différents types d'écrans peuvent être utilisés : des écrans par courant de Foucault qui peuvent fonctionner à la température de l'hélium liquide, à la température de l'azote liquide ou encore à des températures intermédiaires. Un écran de cuivre à la 
température de l'azote liquide est utilisé sur le TOKAMAK T 7 [2].

L'étude T. E. P. R. utilise un écran à $4 \mathrm{~K}$ dans la partie interne du tore et à $18 \mathrm{~K}$ dans la partie externe [5]. Des écrans utilisant des matériaux supraconducteurs peuvent aussi être envisagés.

Dans la fin de ce chapitre, on essaye de donner quelques ordres de grandeur pour situer les problèmes liés à la réalisation des grands TOKAMAKS. L'énergie stockée va de $1,8 \mathrm{GJ}$ pour le J. E. T. à $30 \mathrm{GJ}$ pour la génération suivante de TOKAMAKS et à 220 GJ pour le réacteur cité dans le tableau I. Ces valeurs sont à comparer à l'énergie de $0,8 \mathrm{GJ}$ de la chambre à bulles B. E. B. C. construite pour le C. E. R. N. et terminée en 1972. L'énergie stockée dans les bobines produisant le champ toroïdal est de deux ordres de grandeur supérieure à ce qui s'est fait ; mais plus que la quantité d'énergie, c'est la densité dans le volume de bobinage qui est importante et cette quantité est indépendante de la taille du TOKAMAK.

L'aimant de la chambre à bulles B. E. B. C. a nécessité $3 \mathrm{t}$ de $\mathrm{Nb}-\mathrm{Ti}$. Ce chiffre est à mettre en parallèle avec les quantités de supraconducteurs nécessaires pour les TOKAMAKS du tableau I qui sont de $27 \mathrm{t}$ et de $160 \mathrm{t}$. Ces chiffres sont aussi à confronter avec ceux du doubleur en construction aux Etats-Unis au laboratoire Fermi. Cet accélérateur de $1 \mathrm{~km}$ de rayon a une énergie de $0,4 \mathrm{GJ}$, les aimants sont pulsés, le champ maximal de $4 \mathrm{~T}$ peut être obtenu en quelques secondes. La construction de cet accélérateur nécessitera $37 \mathrm{t}$ de $\mathrm{Nb}-\mathrm{Ti}$. En conclusion, les quantités de supraconducteurs nécessaires, compte tenu des dates de réalisation, ne poseront pas de problème particulier.

Le matériau constituant la structure mécanique de la bobine entre pour une part importante. Les deux études considérées utilisent 1430 t et $7200 \mathrm{t}$ de conducteurs (supraconducteur + cuivre) et respectivement $1590 \mathrm{t}$ et $7230 \mathrm{t}$ d'acier inox pour la structure de la bobine. Dans ces cas, la part du conducteur et celle du matériau de structure de maintien sont égales. Il faut remarquer que le taux de contrainte dans la bobine est indépendant de la taille du réacteur dans certaines hypothèses développées en annexe.

3. Description de l'étude « tore supra ». - Le développement des supraconducteurs pour permettre la réalisation des grands TOKAMAKS nécessite que soient développées, d'une part, des maquettes significatives d'un point particulier de la technologie comme la mesure des pertes dans des champs variables, le comportement thermique de la bobine compte tenu de sa géométrie et du fluide utilisé ou la réalisation des enceintes du cryostat; d'autre part, que soit réalisée une large expérience. Cette expérience ne pouvant être significative que d'un nombre limité de points, différentes voies sont proposées :

- réalisation d'un tore constitué d'un ensemble de bobines,
- réalisation d'une seule grande bobine de la taille de la seconde génération de TOKAMAK,

- réalisation d'un TOKAMAK ayant des bobines supraconductrices.

A la demande du Département de Fusion Contrôlée du Centre de Fontenay-aux-Roses, la version supraconductrice, d'un TOKAMAK utilisant des bobines conventionnelles a été étudiée [6]. C'est cette étude que nous décrivons maintenant [7].

Les principaux paramètres de ce TOKAMAK sont donnés dans le tableau suivant.

\section{TABLEAU II}

Principaux paramètres

$\begin{array}{lll}\text { Grand rayon } & \text { (m) } \ldots & 1,70 \\ \text { Petit rayon } & \text { (m) } \ldots & 0,65 \\ \text { Champ magnétique } & \text { (T) } \ldots & 3 \\ \text { Courant plasma } & \text { (MA) } \ldots & 1,5 \\ \text { Temps d'établissement du courant } & \text { (s) } \ldots . . . & 0,15\end{array}$

La figure 4 montre une coupe de l'ensemble du TOKAMAK ; toutes les contraintes imposées au projet conventionnel sont respectées dans cette étude : citons les 12 sections d'observations comprenant chacune 3 accès au plasma, l'étuvage à $400^{\circ} \mathrm{C}$ de la chambre à vide du plasma, les bobines étant à basse température.

Le conducteur retenu dans cette étude est un câble dont les composites sont isolés à l'oxyde ; le tableau suivant résume les principales caractéristiques de ce conducteur.

\section{TABLEAU III}

Caractéristiques du conducteur

Courant nominal (A) ............

2000

Induction nominale $(\mathrm{T}) \ldots \ldots \ldots \ldots$

Facteur de fonctionnement ..........

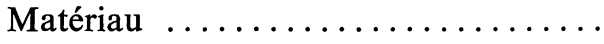

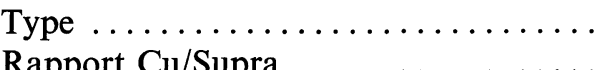

Diamètre des filaments $(\mu) \ldots \ldots \ldots \ldots$

Nombre de filaments ..............

Diamètre du composite $(\mathrm{mm}) \ldots \ldots \ldots$

Pas de torsade $(\mathrm{mm}) \ldots \ldots \ldots \ldots \ldots$

Nombre de composites............

Dimension du câble $\left(\mathrm{mm}^{2}\right) \quad \ldots \ldots \ldots \ldots$

Pas de câblage $(\mathrm{mm}) \ldots \ldots \ldots \ldots \ldots$ Câble plat 3

14

1800

La figure 5 donne la densité d'énergie dissipée le long de la bobine pour un temps d'établissement du champ poloïdal de $0,15 \mathrm{~s}$. La densité d'énergie est $310 \mathrm{~J} / \mathrm{m}^{3}$ au point où le champ est maximal et $1480 \mathrm{~J} / \mathrm{m}^{3}$ au point où les pertes sont maximales [8]. Compte tenu d'un comportement adiabatique, les élévations de température sont respectivement de $0,17 \mathrm{~K}$ et de $1,06 \mathrm{~K}$. Cette élévation de température a 


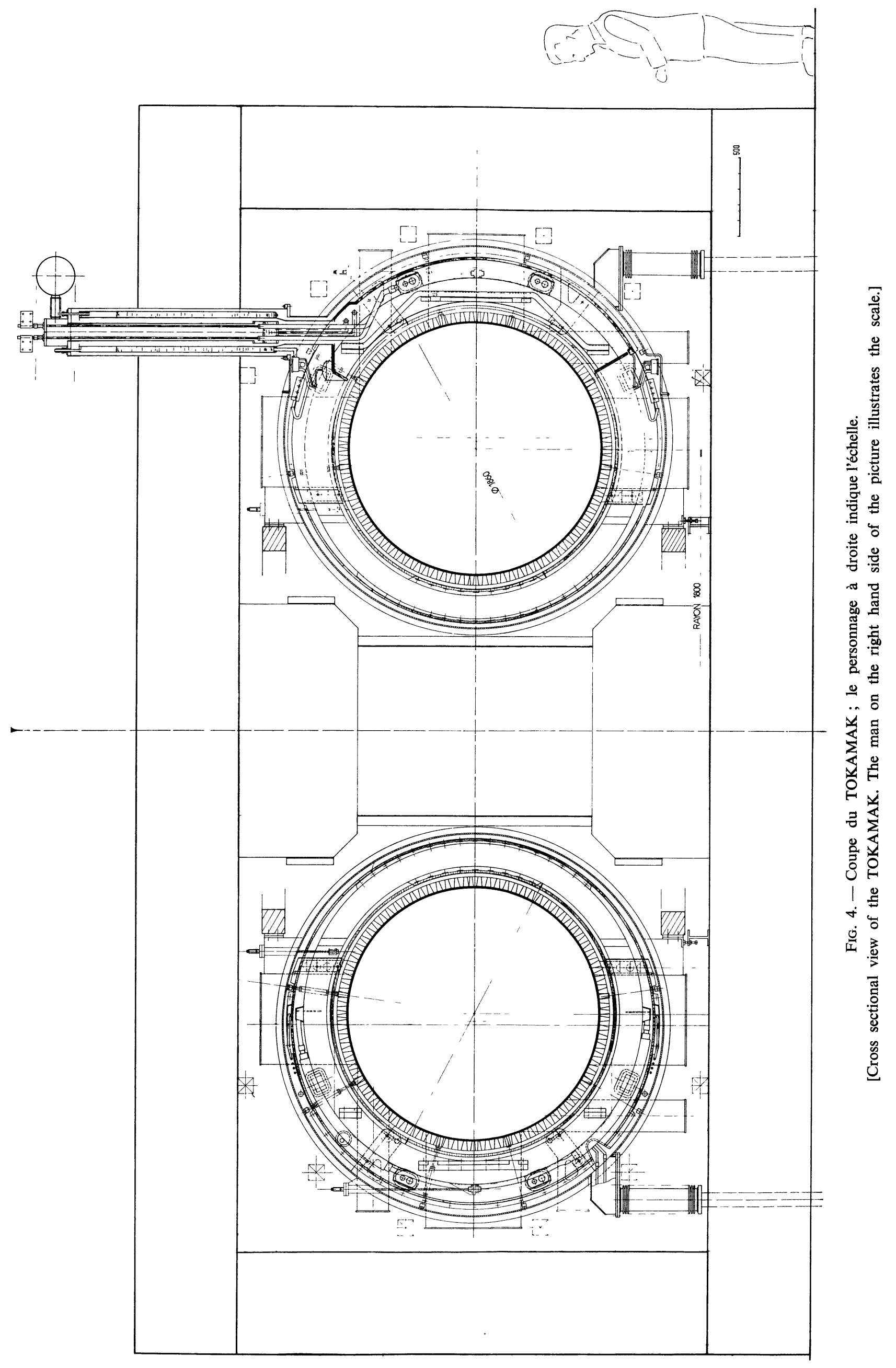




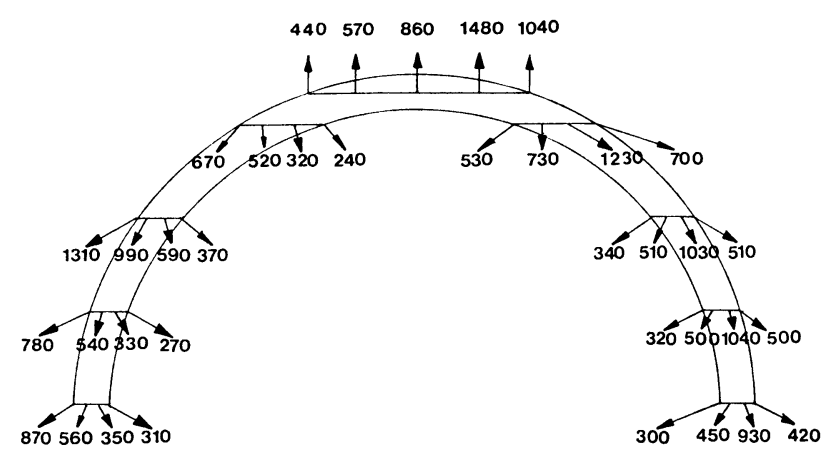

Fig. 5. - Densité d'énergie dissipée en $\mathrm{J} / \mathrm{m}^{3}$ le long de la bobine pour un temps d'établissement du champ poloïdal de $0,15 \mathrm{~s}$.

[Energy density of the losses along the coil for a time constant of the poloïdal field of $0.15 \mathrm{~s}$.]

pour effet de rapprocher le point de fonctionnement de la courbe de courant critique. Le point de fonctionnement passe de 0,79 à 0,83 pour le premier point considéré (champ maximal) et de 0,20 à 0,23 pour le second point (pertes maximales). Pour le point le plus critique du bobinage (point de champ maximal), l'effet de l'établissement du champ poloïdal reste faible. Des temps de montée du champ plus courts ont été considérés sans que la situation devienne très différente ; ceci est dû à la prédominance des pertes par magnétisation au point de champ maximal et ces pertes sont indépendantes du temps de montée.

Le temps de destruction accidentelle du courant plasma est estimé à $20 \mathrm{~ms}$ pour le TOKAMAK étudié, mais la configuration du champ est différente de celle considéré précédemment; les résultats obtenus ne donnent donc qu'une première estimation des effets de la destruction du plasma.

La figure 6 montre la section intérieure d'une bobine. Le bobinage est maintenu par une structure mécanique en acier non magnétique ; cet ensemble soudé contient

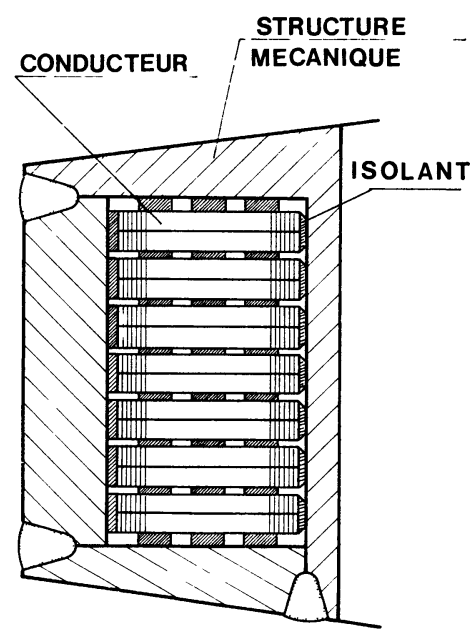

FIG. 6. - Vue schématique de la section intérieure de la bobine montrant le conducteur et la structure mécanique.

[Schematic view of the coil section showing the conductor and the mechanical structure.] l'hélium liquide. Le diamètre d'une bobine est d'environ $2 \mathrm{~m}$, le système magnétique est constitué de 24 bobines et l'énergie magnétique est de $120 \mathrm{MJ}$.

Les bobines sont disposées en voûte. Cette voûte permet de reprendre les forces électromagnétiques. Les couples provenant du champ poloïdal sont contenus par des barres de liaison. Dans ces conditions, toutes les forces électromagnétiques sont reprises à basse température. La figure 7 présente la vue de dessus de l'ensemble du tore.

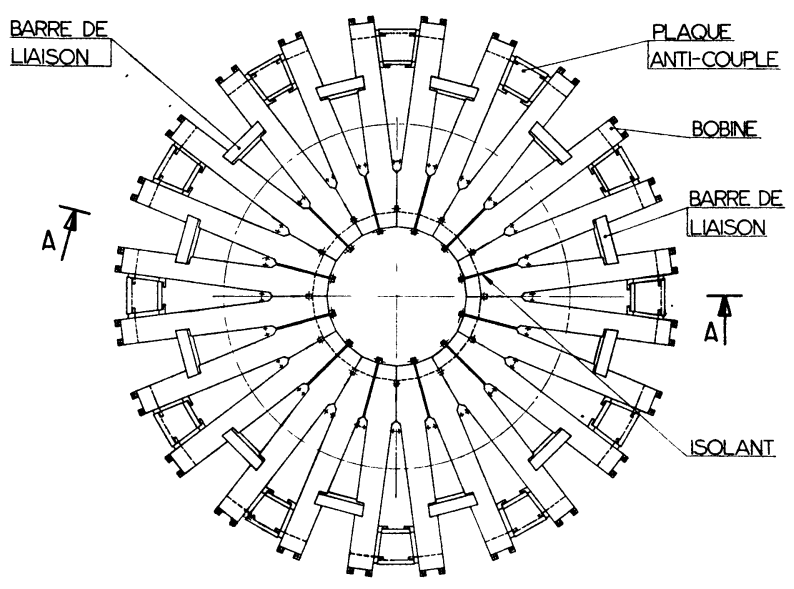

Fig. 7. - Vue de dessus du tore montrant la structure mécanique. [Top view of the torus showing the mechanical structure.]

Le système de réfrigération utilise de l'hélium liquide sous refroidi fonctionnant entre 4 et $4,1 \mathrm{~K}$. Le réfrigérateur a une puissance équivalente à un débit d'hélium liquide de $150 \mathrm{l} / \mathrm{h}$. Le refroidissement du tore est prévu en 120 heures.

Lors du retour accidentel à l'état normal d'une bobine ou d'une fraction de bobine, le système de protection étudié fait transiter l'ensemble des 24 bobines ; dans ces conditions l'énergie dissipée est répartie et la température ne s'élève qu'à $70 \mathrm{~K}$, valeur pour laquelle les déformations dues aux dilatations restent faibles. Le système de réfrigération permet un retour à la température d'équilibre en 15 heures.

Les 24 bobines sont alimentées en série. Une alimentation de $15 \mathrm{~V}, 2000$ A permet d'obtenir le champ nominal en 2 heures.

4. Conclusion. - Il est aujourd'hui évident que la fusion thermonucléaire par la voie TOKAMAK utilisera des bobinages supraconducteurs. D'autre part, là situation de la technologie des supraconducteurs permet dès maintenant d'envisager des réalisations.

L'étude décrite montre la possibilité de satisfaire les contraintes imposées par une expérience de physique tout en utilisant des bobinages supraconducteurs. De plus, les problèmes rencontrés sont très significatifs de ceux posés pour la réalisation des grands TOKAMAKS qui doivent aboutir aux réacteurs. 
Annexe : remarques sur une classe de TOKAMAKS. - Le but est d'essayer de voir comment varient les problèmes posés par la réalisation d'un TOKAMAK lorsque l'on augmente ses dimensions.

DÉfinition D'UNE ClASSE DE TOKAMAKS. Les principales caractéristiques des champs magnétiques d'un TOKAMAK peuvent être décrites à partir de 4 grandeurs :

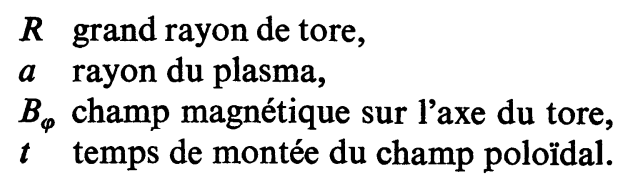

A partir des 3 premières grandeurs, il est possible de connaitre le courant plasma lequel définit une figure de mérite du tore :

$$
I_{\mathrm{p}}=\frac{2 \pi}{\mu_{0}}\left(\frac{B_{\varphi}}{q}\right)\left(\frac{a}{R}\right)^{2} R \quad \text { avec } q=2,5 .
$$

La classe de TOKAMAKS ayant même valeur pour le champ magnétique $B_{\varphi}$ et même valeur pour le rapport d'aspect $R / a$ couvre des machines allant jusqu'au réacteur. Cette classe de machine peut être déterminée par une transformation homothétique sur la géométrie du tore.

Dans ce qui suit, on examine les conséquences de cette transformation sur les principales caractéristiques du TOKAMAK. Les relations données peuvent être souvent déduites de simples considérations sur les dimensions.

Champs magnétiques. - On a vu que le courant plasma varie comme le grand rayon du tore ; on va exprimer en fonction du grand rayon les principales grandeurs caractéristiques des champs magnétiques.

L'énergie stockée dans le champ toroïdal s'exprime par la simple formule :

$$
W=\frac{\pi^{2}}{\mu_{0}}\left(\frac{a_{1}}{R}\right)^{2} B_{\varphi}^{2} R^{3} \quad \mathrm{~d}^{\prime} \text { où } \quad W \propto R^{3}
$$

où $a_{1}$ est le rayon de la bobine.

La valeur maximale du champ magnétique s'écrit :

$$
B_{\max }=\frac{B_{\varphi}}{1-\frac{a_{1}}{R}} k_{\mathrm{d}}
$$

où $k_{\mathrm{d}}$ est un facteur géométrique, d'où $B_{\max }$ constant.

Le prix du matériau supraconducteur est caractérisé par le produit longueur courant qui s'écrit :

$$
\mathcal{L} I=\frac{4 \pi^{2}}{\mu_{0}} B_{\varphi}\left(\frac{a_{1}}{R}\right) R^{2} \quad \text { d'où } \quad \mathcal{L} I \propto R^{2} .
$$

Le champ vertical a pour expression :

$$
B_{\mathrm{v}}=\frac{\mu_{0} I_{\mathrm{p}}}{2 \pi R}\left[\log \frac{8 R}{a}-\frac{3}{2}+\beta+\frac{l_{\mathrm{i}}}{2}\right]
$$

Les quantités $\beta$ et $l_{\mathrm{i}} / 2$ étant indépendantes de la grandeur du TOKAMAK, le champ vertical est lui aussi indépendant de la grandeur du TOKAMAK.

DENSITÉ DE COURANT. - La densité de courant moyenne dans la bobine produisant le champ toroïdal est donnée par la formule suivante où $d$ est le côté de la bobine supposée de section carrée et $N$ le nombre de bobines.

$$
j=\frac{2 \pi}{\mu_{0}} \frac{B_{\varphi}}{N\left(\frac{d}{R}\right)^{2}} \frac{1}{R} \text { d'où } j \propto \frac{1}{R} .
$$

Cette formule montre que la densité de courant moyenne dans la bobine doit varier comme l'inverse du rayon dans la classe de TOKAMAK considérée. Si la grandeur du TOKAMAK augmente, il est possible d'utiliser de faibles densités de courant ; le mode de refroidissement peut être différent, la stabilité cryogénique du conducteur est utilisable.

Une voie différente de celle définie utilisant le même type de conducteur à forte densité aboutit à des impossibilités comme cela sera montré dans le chapitre ASPECT MÉCANIQUE.

Dans l'expression donnant la densité de courant, il est possible d'introduire l'énergie stockée ; on obtient :

$j=\frac{2 \pi}{\mu_{0}} \frac{B_{\varphi}}{N\left(\frac{d}{R}\right)^{2}}\left[\frac{\pi^{2}}{\mu_{0}} \frac{B_{\varphi}^{2}}{W}\left(\frac{a_{1}}{R}\right)^{2}\right]^{1 / 3}$ d'où $j \propto \frac{1}{W^{1 / 3}}$.

On retrouve une loi bien connue sur laquelle s'alignent les réalisations d'aimants supraconducteurs. L'analogie vient du fait que l'on utilise les matériaux à un même niveau de contrainte mécanique.

DENSITÉ D'ÉNERGIE DANS LES BOBINES. - Une quantité importante est le quotient de l'énergie magnétique du champ toroïdal par le volume des bobines. L'énergie stockée a pour expression :

$$
W=\frac{\pi^{2}}{\mu_{0}}\left(\frac{a_{1}}{R}\right)^{2} B_{\varphi}^{2} R^{3}
$$

et le volume des bobines :

$$
v=2 \pi a_{1} d^{2} N
$$

compte tenu du théorème d'Ampère qui s'écrit :

$$
\frac{B_{\varphi}}{\mu_{0}} 2 \pi R=j d^{2} N
$$

on a

$$
v=\frac{4 n^{2}}{\mu_{0}} \frac{a_{1} B_{\varphi} R}{j}
$$

d'où l'expression de la densité d'énergie

$$
\frac{W}{v}=\frac{1}{4}\left(\frac{a_{1}}{R}\right) B_{\varphi} j R
$$


Dans le cas considéré où $j \propto \frac{1}{R}$, la densité d'énergie dans les bobines est indépendante de la grandeur du TOKAMAK. A cette densité d'énergie peut être associée une température ; en considérant des conditions adiabatiques, c'est une approximation de la température prise par la bobine après retour à l'état normal. Cette température caractérise donc les problèmes de protection.

INTENSITÉ DU CONDUCTEUR. - Des considérations sur la protection de la bobine amènent au choix de l'intensité du conducteur. Si l'énergie magnétique échauffe adiabatiquement le conducteur, on a :

en faisant intervenir l'énergie stockée $W=\frac{1}{2} L I_{\mathrm{m}}^{2}$ où $L$ est le self de la bobine et $I_{\mathrm{m}}$ le courant de fonctionnement ; en supposant que la constante de temps de décharge est imposée par la résistance extérieure $R_{\mathrm{ex}}$ et en posant que la tension s'écrit $U_{\mathrm{m}}=R_{\mathrm{ex}} I_{\mathrm{m}}$, on obtient le résultat bien connu :

$$
\frac{j^{2} W}{U_{\mathrm{m}} I_{\mathrm{m}}}=\int_{T_{0}}^{T_{\mathrm{m}}} \frac{c}{\rho} \mathrm{d} t .
$$

La quantité $j^{2} W$ peut s'exprimer en fonction des paramètres du tore en faisant intervenir le rayon $R$ du tore et le volume $v$ des bobines :

$$
j^{2} W=\frac{16 \pi^{2}}{\mu_{0}} R\left(\frac{W}{v}\right)^{2}
$$

d'où la valeur du produit $U_{\mathrm{m}} I_{\mathrm{m}}$ :

$$
U_{\mathrm{m}} I_{\mathrm{m}}=\frac{\frac{16 \pi^{2}}{\mu_{0}} R\left(\frac{W}{v}\right)^{2}}{\int_{T_{0}}^{T} \frac{c}{\rho} \mathrm{d} t}
$$

On a vu que dans l'hypothèse retenue la quantité $\left(\frac{W}{v}\right)$ était indépendante de la dimension du tore, ce qui conduit à avoir le produit $\mathrm{U}_{\mathrm{m}} I_{\mathrm{m}}$ proportionnel au rayon de tore. La tension $U_{\mathrm{m}}$ détermine l'isolement de la bobine ; si l'on admet que cette tension est indépendante de la dimension du tore, le courant est proportionnel au rayon de tore. A un courant de $2000 \mathrm{~A}$ pour TORE SUPRA correspond un courant de $10000 \mathrm{~A}$ pour les machines de grande dimension, valeur généralement retenue.

ASPECT MÉCANIQUe. - Pour aborder le problème sous une forme analytique simple, on considère le cas d'un tore continu d'épaisseur $e$, produisant le champ toroïdal de valeur au centre $B$.

La contrainte maximale sur le tore a pour expression :

$$
\sigma=\frac{B_{\varphi}^{2}}{2 \mu_{0}} \frac{a_{1}}{e} \frac{\log \left(1-\frac{a_{1}}{R}\right)}{\frac{a_{1}}{R}\left(1-\frac{a_{1}}{R}\right)}
$$

En introduisant la densité de courant $j_{\mathbf{i}}$ dans la partie interne du tore,

$$
\frac{B}{\mu_{0}} 2 \pi R=j_{\mathrm{i}} 2 \pi\left(R-a_{1}\right) e
$$

on obtient :

$$
\sigma=\frac{B_{\varphi} j_{\mathrm{i}} R}{2} \log \left(1-\frac{a_{1}}{R}\right) .
$$

Compte tenu de la relation sur la densité de courant $j_{\mathbf{i}} \propto 1 / R$, la contrainte est indépendante de la dimension du tore. On voit que l'utilisation d'un même conducteur conduirait à avoir des contraintes proportionnelles à la dimension du tore.

De même la force de centrage sur les bobines qui peut s'écrire :

$$
F_{x}=\frac{\pi}{\mu_{0}} B_{\varphi}^{2} R^{2}\left(\frac{a_{1}}{R}\right)^{2}
$$

conduit à des contraintes indépendantes de la taille du tore. La même remarque est valable pour le couple produit par le champ vertical.

$$
C=\frac{2 \pi^{2}}{\mu_{0}}\left(\frac{a_{1}}{R}\right) \frac{B_{\mathrm{v}} B_{\varphi}}{N} R^{3} .
$$

On peut remarquer en comparant la formule donnant la densité d'énergie dans la bobine $(W / v)$ et la formule donnant la contrainte dans le tore continu, la relation simple entre ces deux quantités.

TEMPS DE MONTÉE DU CHAMP POLOÏDAL. - Le temps de montée du champ poloïdal joue un rôle fondamental dans l'étude des éléments d'un tore supraconducteur (conducteur et source d'énergie des bobines), malheureusement il ne peut entrer dans un formalisme simple.

On peut peut-être dire que la constante de temps de montée du courant serait proportionnelle au grand rayon de tore, ce qui assure que la vitesse de montée du courant plasma au temps zéro serait constante. Le champ vertical étant indépendant de la taille du tore, la vitesse de variation du champ magnétique serait proportionnelle à $1 / R$.

CONCLUSIONS SUR LA FAMILLE DE TOKAMAK CONSIDÉRÉE. - Pour la classe de machines considérée, machines à $B_{\varphi}$ constant et géométriquement homothétique, on a les variations suivantes en fonction du grand rayon $R$.

Energie stockée .............. $R^{3}$

Quantité de supraconducteur ........ $R^{2}$

Champ vertical ............................. $R^{0}$

Densité de courant ..................... $R^{-1}$

Densité d'énergie dans la bobine ....... $R^{0}$

Produit $U_{\mathrm{m}} I_{\mathrm{m}} \ldots \ldots \ldots \ldots \ldots \ldots \ldots \ldots \quad R^{1}$

Contraintes mécaniques ........... $R^{0}$

Vitesse de montée du poloïdal ....... $\approx R^{-1}$ 
L'étude d'un tore de petite taille permet d'obtenir des résultats très significatifs sur les problèmes des contraintes mécaniques, sur la protection des bobines et sur l'ensemble des problèmes de réalisation. Le conducteur est nécessairement différent suivant la taille de la machine considérée. Le tore de petite dimension nécessite un conducteur à forte densité qui subit des variations plus rapides des champs extérieurs.

\section{Bibliographie}

[1] LeLoup, C., Aspects techniques d'un réacteur thermonucléaire à confinement magnétique, Congrès National de Physique des Plasmas, 6/10 décembre 1976, Paris.

[2] Ivanov, D. P., Testing results of " TOKAMAK 7 " Superconducting magnet system (SMS) sections, Applied Superconductivity Conference, 17/20 August 1976, Stanford.

[3] Martinelli, A. P., Calcul d'un écran, Département SATURNE/SEDAP/75-166, décembre 1975, CEN/Saclay.

[4] TURCK, B., Experimental and theoretical approach of current distribution and losses in superconducting composites for fusion magnets, Applied Superconductivity Conference, 17/20 August 1976, Stanford.

[5] TOKAMAK experimental power reactor conceptual design, ANL/CTR, 76-3, August 1976, Stanford.

[6] TORUS, II, Technical description of the design proposal EUR CEA FC 824. June 1976.

[7] Etude de bobines supraconductrices produisant le champ toroidal d'un TOKAMAK expérimental. Version supraconductrice de TORE II, DPh/PE, STIPE/76-72, octobre 1976, CEN/Saclay.

[8] Genevey, P., Pertes induites dans le conducteur «TORE SUPRA », DPh/PE, STIPE 76-84, novembre 1976, CEN/Saclay. 